\section{S2A:5 DEVELOPMENT AND VALIDATION OF A SCORE TO PREDICT THE RISK OF SEVERE INFECTION IN SLE}

'B Segura, ${ }^{2}$ Rúa-Figueroa, ${ }^{3} \mathrm{JM}$ Pego-Reigosa, ${ }^{4} \mathrm{~V}$ Del Campo, ${ }^{5} \mathrm{D}$ Isenberg, ${ }^{5} \mathrm{~A}$ Rahman. ${ }^{1}$ Insular University Hospital, Rheumatology Department, Las Palmas de Gran Canaria, Spain; ${ }^{2}$ Doctor Negrín University Hospital, Rheumatology Department, Las Palmas de Gran Canaria, Spain; ${ }^{3}$ Biomedical Research Institute of Vigo (IBIV), University Hospital Complex of Vigo, Rheumatology Department, Vigo, Spain; ${ }^{4}$ Biomedical Research Institute of Vigo (IBIV), University Hospital Complex of Vigo, Preventive Medicine Department, Vigo, Spain; ${ }^{5}$ University College London Hospital, Centre for Rheumatology, Department of Medicine, London, UK

10.1136/lupus-2018-abstract.8

Purpose To develop a predictive risk score that assesses the probability of severe infection in SLE patients and to test it in an independent cohort.

Methods The SLE severe infection score (SLESIS) was developed using data from the RELESSER (Spanish Society of Rheumatology Lupus Registry) cohort of 3658 SLE patients using a Cox regression model for repeated events (AndersenGill) The results were expressed as hazard ratio (HR) of developing one serious infection/1000 patient-years for patients with the risk factor compared to those without that factor. SLESIS for an individual patient is the sum of the HR values of all factors present at that time.

SLESIS was validated using retrospective data from the UCLH (University College London Hospital) cohort including 699 SLE patients.

Results The risk factors included in SCORE and their HR calculated from RELESSER data are shown in table 1. From 699 SLE UCLH patients, 98 (14\%) developed serious infection.

\section{Abstract S2A:5 Table 1}

\begin{tabular}{|l|l|l|l|}
\hline Risk factor & B & P-value & HR \\
\hline $\begin{array}{l}\text { Age at diagnosis } \\
\text { (>46 years old) }\end{array}$ & 0.1163 & 0.001 & 1.12 \\
\hline $\begin{array}{l}\text { Latin American } \\
\text { ethnicity }\end{array}$ & 0.427 & 0.001 & 2.40 \\
\hline $\begin{array}{l}\text { Corticosteroids } \\
\text { (>10 me/day) at } \\
\text { time } \\
\text { calculating scorE } \\
\text { of }\end{array}$ & 0.2878 & 0.001 & 1.33 \\
\hline $\begin{array}{l}\text { Sex=male } \\
\text { hospitalization } \\
\text { (for SLE) }\end{array}$ & 1.0049 & $<0.00001$ & 2.73 \\
\hline $\begin{array}{l}\text { Katz index } \\
\text { any time }\end{array}$ & 0,062 & 0.002 & 1.06 \\
\hline $\begin{array}{l}\text { Prior infection at } \\
\text { Previous }\end{array}$ & 0.8739 & $<0.0001$ & 2.40 \\
\hline
\end{tabular}

We compared these patients with 111 SLE controls who never suffered serious infection. The characteristics of the SLE infection and SLE non-infection groups are summarised in table 2.

Median SLESIS at diagnosis in patients with infection was 4.27 (IQR 3.18) which was significantly higher than in the control group (Median 2.55, IQR 3.79) ( $\mathrm{z}=3.341 ; \mathrm{p}=0.0008$ ). Median SLESIS just before infection was 5.3 (IQR 3.68) which was significantly higher compared to SLESIS at diagnosis $(\mathrm{z}=-5.733 ; \mathrm{p} \leq 0.001)$ in those patients or SLESIS measured at the same time post-diagnosis in the non-infected group (Median 3.73 RI 3.7) ( $\mathrm{z}=-4.765, \mathrm{p} \leq 0.001)$.

By Receiver Operator Characteristic analysis, we defined three possible cut-offs to distinguish patients with and without infection. For SLESIS just before infection, the area under the ROC curve was 0.75 (CI: 0.66 to 0.84 ) and the three selected cut offs $(3.67,3.79,4.24)$ reached a sensitivity of $90 \%$ and specificity of $50 \%$.

Conclusion We have developed a score for predicting risk of serious infection in SLE and validated it in an independent cohort. Given the potential mortality from such infections, SLESIS could be clinically useful though the moderate sensitivity and specificity necessitate caution and further prospective studies.

\section{S2A:6 SOLUBLE UROKINASE PLASMINOGEN ACTIVATOR RECEPTOR (SUPAR) PREDICTS THE DEVELOPMENT OF ORGAN DAMAGE OVER 5 YEARS IN SYSTEMIC LUPUS ERYTHEMATOSUS: RESULTS FROM THE SLICC INCEPTION COHORT}

${ }^{1} \mathrm{H}$ Enocsson, 'L Wirestam, ${ }^{1} \mathrm{~J}$ Wetterö, ${ }^{1} \mathrm{~T}$ Skogh, The Slicc Group2 ${ }^{2},{ }^{3,4} \mathrm{IN}$ Bruce, ${ }^{1} \mathrm{C}$ Sjöwall. ${ }^{1}$ Rheumatology, Division of Neuro and Inflammation Sciences,Department of Clinical and Experimental Medicine Linköping Uni, Linköping, Sweden; ${ }^{2}$ The Systemic Lupus International Collaborating Clinics (SLICC) Group; ${ }^{3}$ Arthritis Research UK Centre for Epidemiology, Centre for Musculoskeletal Research, The University of Manchester, Manchester, UK; ${ }^{4}$ NIHR Manchester Musculoskeletal Biomedical Research Unit, Central Manchester University Hospitals NHS Foundation Trust, Manchester, UK

\subsection{6/lupus-2018-abstract.9}

Background The urokinase plasminogen activator receptor (suPAR) participates in proteolysis, migration and adhesion. Receptor shedding yields a soluble form (suPAR) that has emerged as a promising severity biomarker in malignancies, inflammatory and infectious diseases. Previously, suPAR was shown to reflect accumulated organ damage in systemic lupus erythematosus (SLE). Here, we investigate suPAR as a potential predictor of future organ damage in patients with recent-onset SLE.

Methods 345 SLE cases (at least 4 ACR criteria) from North America, Europe and Asia were included. All patients were from the SLICC inception cohort and were selected based on a minimum of 5 years follow-up and absence of organ damage (SLICC/ ACR damage index; SDI $>0$ ) at inclusion. Patients were enrolled within 15 months of diagnosis. Estimated glomerular filtration rate (eGFR) was available for 180 patients. Serum suPAR levels were measured by ELISA at inclusion only, and levels were related to SDI after 5 years of follow-up. Age- and sex-matched controls $(1: 1)$ were from the Swedish population. 